\title{
The Viscosity Behavior of ABA-Type Block Copolymer Composed of 2-Hydroxyethyl Methacrylate and Styrene in Organic Solvent Mixture
}

\author{
Teruo OKano, Masahisa IKemi, and Isao Shinohara \\ Department of Polymer Chemistry, Waseda University, \\ Nishiohkubo, Shinjuku-ku, Tokyo 160, Japan.
}

(Received July 25, 1977)

\begin{abstract}
The ABA-type block copolymer and the random cooligomer composed of a hydrophilic monomer, 2-hydroxyethyl methacrylate (HEMA), and a hydrophobic monomer, styrene (St), were synthesized to study the influence of the hydrophilic and the hydrophobic block chains on the polymer properties in dilute solution. Viscometric measurements were carried out at various temperatures in two solvent mixtures composed of $\mathrm{N}, \mathrm{N}$ dimethylformamide (DMF) or ethanol and dioxane which had preferential affinity to one of the block chains. It was found by viscometric measurements that the solution behavior of the ABA-type block copolymer containing a 0.394-mol fraction of HEMA was remarkably different from that of the random cooligomer of the same composition. That is, in each polymer system, the viscosity curves showed maximum values at an optimum composition of the solvent mixture. The peak for the block copolymer, however, shifted to the DMF- or ethanol-rich composition at any one temperature in comparison with that for the random cooligomer. The shift of the peak became large with decreasing temperature. In DMFdioxane system, especially, the intrinsic viscosity vs. solvent composition curve displayed a cusp at about 0.4 -vol fraction of dioxane, suggesting that a conformational transition took place within the block copolymer chain. The cusp smoothed out with decreasing temperature, and changed into a maximum appearing at about 0.2-vol fraction of dioxane.

KEY WORDS 2-Hydroxyethyl Methacrylate / Styrene / Telomerization / Telechelic Oligomer / Intrachain Phase Separation / Intrinsic Viscosity / Block Copolymer /
\end{abstract}

Polymers consisting of both the hydrophilic and the hydrophobic portion have characteristic properties, often referred to as the amphiphilic property. However, only a few papers have been published on the relation between the polymer structure and the amphiphilic property. The conditions for the occurrence of either the hydrophilic or the hydrophobic functions still remain unclarified and so it seems desirable to study the influence of intramolecular interaction on the solution properties more extensively.

It has been reported that the hydrophilic and the hydrophobic functional occurrences of the amphiphilic polymer were influenced not only by the chemical structure and the composition of hydrophilic and hydrophobic groups but also by their distribution along the chain. ${ }^{2-5)}$ Consequent- ly, the block copolymer composed of the hydrophilic and the hydrophobic chain is considered to be a good model for investigating the influence of the molecular constitution on solution behavior.

The study on the conformation and intramolecular interaction of block copolymers consisting of an incompatible homopolymer pair in dilute solution has been the subject of extensive investigation. ${ }^{6)}$ It is known that block copolymers adopt an intrachain phase-separated structure under adequate conditions. However, a unified conclusion has not been obtained as yet for the proposed structures of the block copolymers.

In this paper, the ABA-type block copolymer and the random cooligomers composed of 2-hydroxyethyl methacrylate (HEMA) and styrene (St) were synthesized and viscometric measure- 
ments were carried out at various temperatures in two solvent mixtures. The difference in solution behavior between the block copolymer and the random cooligomer is discussed with respect to the molecular constitution.

\section{EXPERIMENTAL}

\section{Purification of Materials}

$\alpha, \alpha^{\prime}$-Azobisisobutyronitrile (AIBN), 2-aminoethanethiol (AESH), 1-buthanethiol (BuSH), $N, N$-dimethylformamide (DMF), and $\mathrm{St}$ were purified by conventional methods. HEMA was distilled under a reduced pressure of nitrogen atmosphere and the fraction of bp $87^{\circ} \mathrm{C}(5 \mathrm{mmHg})$ was used. Dioxane was first distilled after refluxing in the presence of hydrochloric acid, and then redistilled after dehydration by metallic sodium for $24 \mathrm{hr}$. Ethanol was distilled at first, and then redistilled after refluxing in the presence of magnesium and a small amount of iodide.

\section{HEMA-St Cooligomerization}

The DMF solution containing the desired amounts of AIBN, HEMA, St, and BuSH was put into each ampoule. Each ampoule was sealed under a vacuum less than $10^{-5} \mathrm{mmHg}$ after degassing three times. The sealed ampoules were immersed in an oil bath at $60^{\circ} \pm 0.1^{\circ} \mathrm{C}$ and shaken vigorously. After the proper reaction time elapsed, the ampoules were removed from the bath and immediately frozen in liquid nitrogen. They were warmed sufficiently to melt contents which were then poured into a ether-petroleum ether mixture to precipitate cooligomers; these were filtered off, washed thoroughly with the precipitant mixture and dried in vacuo.

Synthesis of Amino-semitelechelic Oligo-HEMA

A DMF solution containing the desired amounts of AIBN, HEMA, and AESH to act as a chaintransfer agent, was put into an ampoule. The ampoule was sealed by conventional methods and immersed in an oil bath at $60.0^{\circ} \pm 0.1^{\circ} \mathrm{C}$ for a given period of time. The contents were then poured into ether to precipitate the oligomer. The resulting oligomer was filtered off and dried in vacuo.

\section{Synthesis of Isocyanate-telechelic Oligo-St}

The desired amounts of St and bis ( $p$-isocyanato- phenyl) disulfide $\left(\right.$ IPDS) ${ }^{7}$ were weighed into a quarz ampoule which was then irradiated with ultraviolet light (Toshiba SHL 100 UV) for a certain period from a distance of $15 \mathrm{~cm}$. The polymerized content was diluted with benzene and poured into hexane to precipitate the isocyanate -telechelic oligo-St. The obtained oligomer was. dried in a vacuum desicator.

Synthesis of HEMA-St ABA-Type Block Copoly-
mer

With $\left[\mathrm{NH}_{2}\right] /[\mathrm{NCO}]=1.2$, the amino-semitelechelic oligo-HEMA (prepolymer $A$ ) and the isocyanate-telechelic oligo-St (prepolymer B) were allowed to react with each other while stirring in the DMF solvent at $0^{\circ} \mathrm{C}$ for $24 \mathrm{hr}$. The reactant was diluted with DMF and poured into methanol-2-propanol mixture. The precipitated sample was filtered off and washed thoroughly with ether. The sample obtained was then dried in a vacuum desiccator.

\section{Analysis of Oligomers and Copolymer}

The polymer structures were confirmed by infrared spectroscopy (Hitachi EPI G-III type). The number-average molecular weight $\left(\bar{M}_{n}\right)$ of the oligo-HEMA was determined by a vaporpressure osmometer (Hitachi V.P.O. 117 type) in methanol solvent. The molecular weight of oligo-St was determined from the viscosity equation. $^{8}$ The amino group concentration was determined by the titration of acetic acid solution of amino-semitelechelic oligo-HEMA with perchloric acid-acetic acid solution using crystal violet as an indicator. ${ }^{9}$ The isocyanate group concentration was determined by back-titration with $\mathrm{HCl}$-methanol solution using bromophenol blue as the indicator, following the reaction of a given concentration of dibutylamine-chlorobenzene solution with isocyanate-telechelic oligoSt for $24 \mathrm{hr}$ at room temperature. ${ }^{10}$

\section{Viscometric Measurement}

The intrinsic viscosity was measured with a modified Ubbelohde viscometer in a thermostatically controlled reservoir.

\section{RESULTS AND DISCUSSION}

\section{Synthesis of the HEMA-St ABA-Type Block Copolymer}

The results of the oligomerization of HEMA 
Viscosity Behavior of HEMA-St ABA Type Block Copolymer

Table I. Preparation and analysis of prepolymers

\begin{tabular}{ccccccc}
\hline Prepolymer & {$[\mathrm{S}] /[\mathrm{M}]$} & $\begin{array}{c}\text { Time, } \\
\mathrm{hr}\end{array}$ & $\begin{array}{c}\text { Conversion, } \\
\%\end{array}$ & $\begin{array}{c}\text { Molecular weight } \\
\text { from analysis of } \\
\text { end group }\end{array}$ & $\bar{M}_{n}$ & $\begin{array}{c}\text { Number of } \mathrm{NH}_{2} \text { or } \\
\text { NCO groups in one } \\
\text { molecule }\end{array}$ \\
\hline $\mathrm{A}^{\mathrm{a}}$ & 0.30 & 1.5 & 7.98 & 3,360 & $3,420^{\mathrm{b}}$ & 1.02 \\
$\mathrm{~B}^{\mathrm{c}}$ & 0.03 & 12.0 & 40.20 & 7,100 & $7,250^{\mathrm{a}}$ & 2.04 \\
\hline
\end{tabular}

a A: oligo-HEMA, $[\mathrm{M}]=2.5 \mathrm{~mol} / l$ in $\mathrm{DMF}$ at $60^{\circ} \mathrm{C}$; $[\mathrm{AIBN}]=5 \times 10^{-3} \mathrm{~mol} / \mathrm{l}$.

b V.P.O. in methanol at $40^{\circ} \mathrm{C}$.

c B: oligo-St, bulk system at $30^{\circ} \mathrm{C}$.

d $[\eta]=3.64 \times 10^{-4} \bar{M}^{0.84}$ (ref 8$)$.

with AESH as a chain-transfer agent and the photooligomerization of St with IPDS as an initiator are shown in Table I. The amino-semitelechelic oligo-HEMA (prepolymer A) could be obtained by the oligomerization of HEMA with AESH as a chain-transfer agent since the oligomerization proceeded according to telomerization mechanism. ${ }^{3,4}$ The molecular weight calculated from the amino-group analysis (one amino group is contained in one oligo-HEMA molecule) was approximately equal to that determined from the V.P.O. measurement. Photooligomerization of St with IPDS as an initiator was carried out to obtain the isocyanate-telechelic oligo-St (pre- polymer B). The molecular weight calculated from the isocyanate-group analysis (two isocyanate groups are contained in one oligo-St) was approximately equal to that determined from the viscosity equation.

Block copolymers of the confirmed structure could be synthesized by the method of block copolymerization using telechelic oligomers as the prepolymer. In order to synthesize HEMA-St ABA-type block copolymer, prepolymer A was allowed to react with prepolymer $B$ in DMF solution at $0^{\circ} \mathrm{C}$ for $48 \mathrm{hr}$. Prepolymer A was soluble in methanol-2-propanol mixture (volume ratio: $1 / 1)$ and insoluble in ether while prepolymer

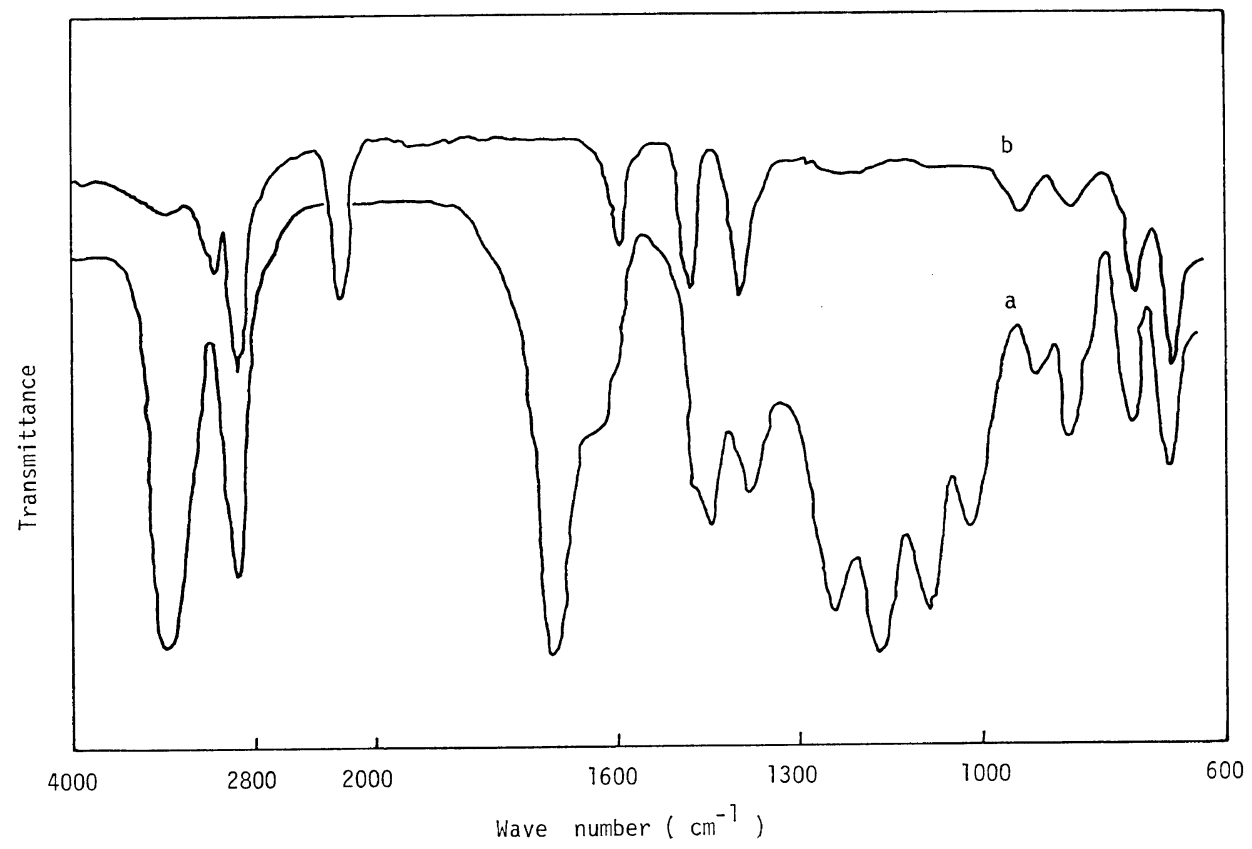

Figure 1. IR spectra of the HEMA-St ABA-type block copolymer and the isocyanate-telechelic oligo-St: a, HEMA-St ABA-type block copolymer; b, isocyanate-telechelic oligo-St. 
B was soluble in ether and insoluble in methanol2-propanol mixture. The purification of the ABA-type block copolymer could be performed in two steps because of the different solubilities of these prepolymers. In the first steps, the unreacted prepolymer A was removed by precipitating the reaction mixture into a methanol-2-propanol mixture (volume ratio: $1 / 1$ ). The precipitate obtained was filtered out. In the second step the unreacted prepolymer $\mathbf{B}$ was removed by thoroughly washing the precipitate with ether.

Figure 1 shows the IR spectra of the ABAtype block copolymer and the oligo-St. The presence of HEMA was demonstrated by the carbonyl band at $1740 \mathrm{~cm}^{-1}$ and many other absorptions also contained in the ABA-type block copolymer. St is also contained in the ABA-type block copolymer as indicated by the aromatic peak at $700 \mathrm{~cm}^{-1}$. The isocyanate group is contained in oligo-St as indicated by the peak at $2270 \mathrm{~cm}^{-1}$; this peak, however, disappears in the ABA-type block copolymer.

Results of the ABA-type block copolymer analysis are shown in Table II. The HEMA mole fraction in the ABA-type block copolymer calculated from the ratio prepolymer $A$ : prepolymer $B=2: 1$ was approximately equal to that determined from the elemental analysis. It has been demonstrated that the microstructure of the block copolymer synthesized by this method is remarkably different from that of the blendmer of oligo-HEMA and oligo-St. ${ }^{3}$

Table II. HEMA-St ABA-type block copolymer ${ }^{\mathrm{a}}$

\begin{tabular}{ccccc}
\hline \multicolumn{2}{c}{ HEMA mol fraction } & & \multicolumn{3}{c}{ Block chain length, $\bar{M}_{n}$} \\
\cline { 1 - 1 } \cline { 1 - 1 } Found & Calcd & & A Block & B Block \\
\hline 0.394 & 0.426 & 3,360 & 7,100 \\
\hline${ }^{\text {a }}\left[\begin{array}{llll}{\left[\mathrm{NH}_{2}\right] /[\mathrm{NCO}]=1.2} \\
\text { time, } 48 \mathrm{hr} \text {; reaction temp, } 0^{\circ} \mathrm{C} .\end{array}\right.$
\end{tabular}

\section{Viscosity Behavior of the HEMA-St Random Cooligomers in Mixed Solvent}

Table III shows the results of HEMA-St cooligomerization with $\mathrm{BuSH}$ as a chain-transfer agent.

Viscometric measurements were carried out on the HEMA-St random cooligomers in DMFdioxane mixture at $30^{\circ} \mathrm{C}$ and these results are
Table III. HEMA - St cooligomerization ${ }^{\text {a }}$

\begin{tabular}{crcc}
\hline $\begin{array}{c}\text { HEMA mol } \\
\text { fraction } \\
\text { in monomer }\end{array}$ & $\begin{array}{c}\text { Time, } \\
\mathrm{hr}\end{array}$ & $\begin{array}{c}\text { Conversion, } \\
\%\end{array}$ & $\begin{array}{c}\text { HEMA mol } \\
\text { fraction } \\
\text { in cooligomer }\end{array}$ \\
\hline $0^{\mathrm{a}}$ & 23.0 & 22.0 & 0 \\
0.2 & 15.0 & 13.6 & 0.374 \\
0.5 & 15.0 & 28.2 & 0.642 \\
0.8 & 5.0 & 23.2 & 0.861 \\
\hline
\end{tabular}

${ }^{a}\left[\right.$ AIBN] $=5 \times 10^{-3} \mathrm{~mol} / l$; [Monomer] $=3 \mathrm{~mol} / l$ in DMF at $60^{\circ} \mathrm{C}$ (a, $2.38 \mathrm{~mol} / l$ in tetrahydrofuran); $[\mathrm{S}] /[\mathrm{M}]=0.02(\mathrm{a}, 0.0033) ; \mathrm{S}, n$-BuSH.

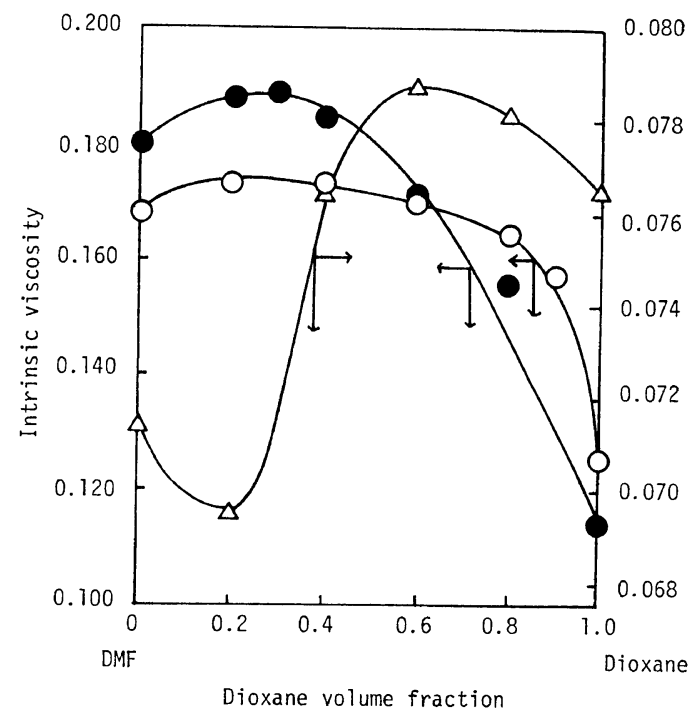

Figure 2. Relation between the intrinsic viscosity of the HEMA-St random cooligomers and the solvent composition in the DMF-dioxane mixture. HEMA mol fraction: $\bigcirc, 0.861 ; \bullet, 0.642 ; \triangle, 0.374$.

illustrated in Figure 2. It was observed that the viscosity curves of the random cooligomers showed a maximum, which shifted to the dioxane-rich composition with increasing St composition of the random cooligomers. Dioxane is a good solvent for oligo-St, though a poor solvent for oligoHEMA. Therefore, it is considered that the molecular chain of the random cooligomers expands with increasing dioxane content in the solvent mixture, as dioxane has a preferential affinity to St units. The molecular chain of the random cooligomers contracts beyond a certain dioxane content, because HEMA units have less affinity to dioxane. This shows that the random 
cooligomers take on the most extended conformation in the solvent mixture with an adequate mixing ratio. Consequently, as the St-rich random cooligomer has a higher affinity to dioxane-rich solvent, the position of the maximum value is shifted to the higher dioxane content with increasing St composition.

The viscosity behavior of the random cooligomer containing $0.374 \mathrm{~mol}$ fraction of HEMA was particularly different from the others, i.e., the viscosity curve showed not only a maximum, as plotted against the solvent composition, but also a minimum at about 0.2 vol fraction of dioxane. The appearance of this minimum suggests that the affinity of the solvent mixture, with a composition of about 0.2 , to the molecular chain of the random cooligomer is considerably suppressed due to the relatively strong interaction between DMF and dioxane. ${ }^{11}$

\section{The Influence of the Molecular Constitution on the Viscosity Behavior}

Figure 3 shows the relation between the intrinsic

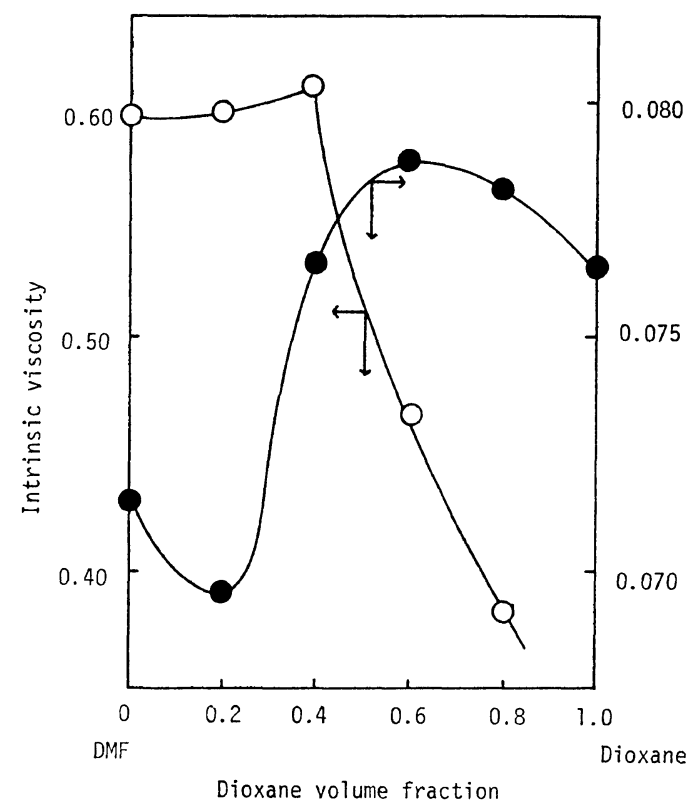

Figure 3. Relation between the intrinsic viscosity and the solvent composition in DMF-dioxane mixture at $30^{\circ} \mathrm{C}$; O, HEMA-St ABA-type block copolymer (HEMA mol fraction, 0.394); $\bullet$, random cooligomer (HEMA mol fraction, 0.374). viscosity and solvent composition obtained for solutions of the HEMA-St ABA-type block copolymer containing a $0.394 \mathrm{~mol}$ fraction of HEMA in DMF-dioxane mixtures at $30^{\circ} \mathrm{C}$. The corresponding relation for the random cooligomer with approximately the same composition is also reproduced from Figure 2 for the sake of comparison. The random cooligomer is soluble in dioxane, while the block copolymer is insoluble. The viscosity curve of the random cooligomer displays a broad peak at about a 0.6 vol fraction of dioxane. However, the viscosity curve of the block copolymer displays a cusp at about a 0.4 vol fraction of dioxane. This contrast seems attributable to the difference in the molecular constitution between these two polymers.

The results of viscometric measurements of the oligo-St in DMF-dioxane mixture are shown in Figure 4. The intrinsic viscosity of the oligoSt monotonously increased with increasing dioxane content. However, the dependence of the intrinsic viscosity on temperature was influenced by the solvent composition in DMF-dioxane mixture. With rising temperature, the intrinsic viscosity of the oligo-St increased in DMF, but decreased in dioxane. In a solvent mixture whose dioxane

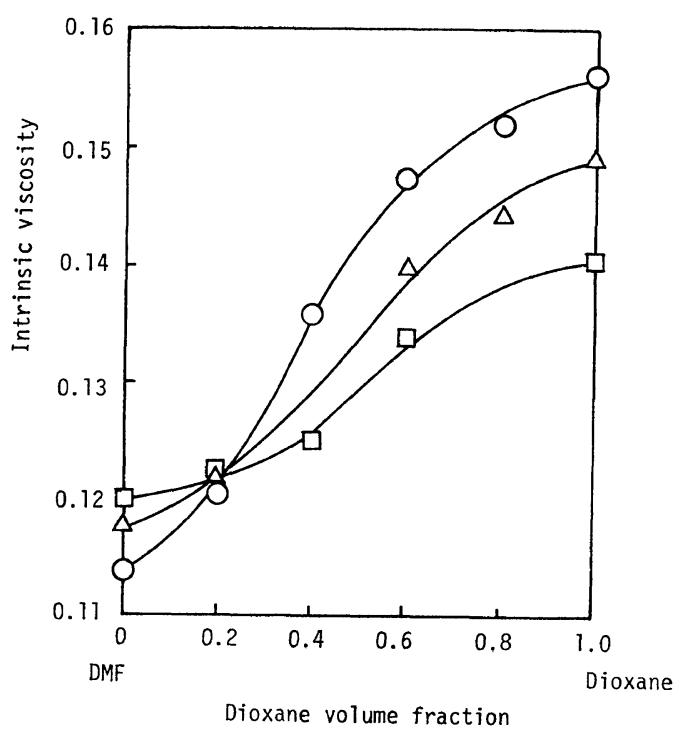

Figure 4. Relation between the intrinsic viscosity of oligo-St and the solvent composition in DMFdioxane mixture: $\bigcirc$, at $15^{\circ} \mathrm{C} ; \triangle$, at $30^{\circ} \mathrm{C} ; \square$, at $50^{\circ} \mathrm{C}$. 
composition was about a 0.2 vol fraction, the intrinsic viscosity was independent of the temperature.

These results show that the molecular chain of the oligo-St adopts a contracted conformation in DMF and an expanded conformation in dioxane. On the contrary, the molecular chain of the oligoHEMA greatly expands in DMF and contracts with increasing dioxane composition. Consequently, DMF and dioxane may be regarded as preferential solvents for the HEMA chain and St chain, respectively.

Figures 5 and 6 show the viscosity behavior of the random cooligomer and the block copolymer in the DMF-dioxane mixture at various temperatures. In both systems, the viscosity curves showed a maximum at the optimum composition of the solvent mixture. In the random cooligomer system, the intrinsic viscosity decreased with increasing temperature over the entire range of the composition, but showed a similar dependence on the solvent composition irrespective of temperature; the position of the maximum, for example, did not shifted. In the block copolymer system, on the contrary, the shape of the viscosity curves,

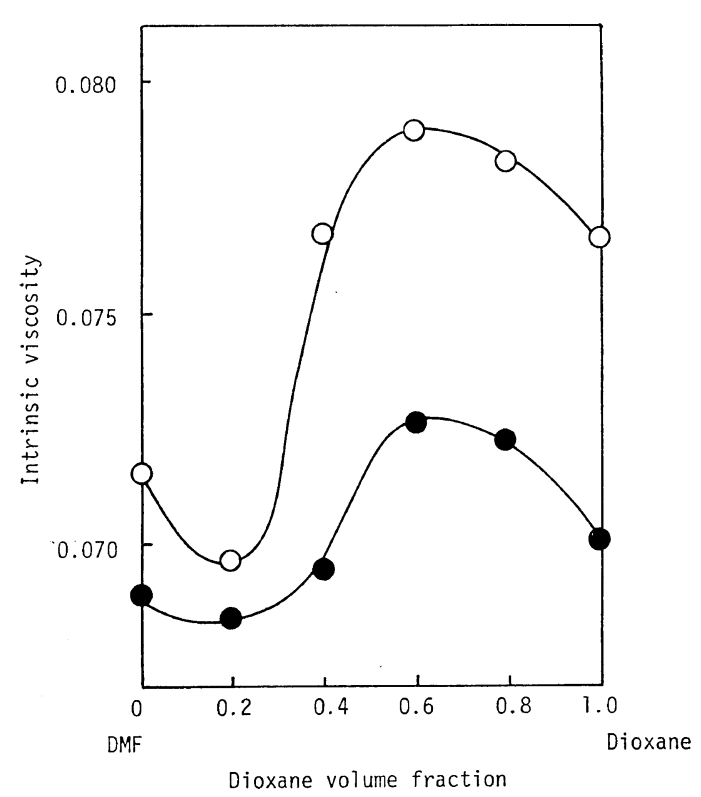

Figure 5. Relation between the intrinsic viscosity of the HEMA-St random cooligomer (HEMA mol fraction, 0.374) and the solvent composition in the DMF-dioxane mixture: $\bigcirc$, at $30^{\circ} \mathrm{C}$; $\bullet$, at $50^{\circ} \mathrm{C}$. different from that of the random cooligomer, exhibited a remarkable temperature dependence. At $15^{\circ} \mathrm{C}$, the viscosity curve of the block copolymer possessed a broad peak at about 0.2 vol fraction of dioxane and the intrinsic viscosity drastically decreased with increasing dioxane composition beyond about a 0.4 vol fraction. With increasing temperature, the peak at about 0.2 disappeared and a sharp transition point appeared at about 0.4 .

In the block copolymer system, the peak shifted to the DMF-rich composition for any one temperature in contrast to the random cooligomer which possessed a maximum at about $0.6 \mathrm{vol}$ fraction. At $15^{\circ} \mathrm{C}$, this peak position of the block copolymer shifted to about 0.2 , which corresponds to that of the random cooligomer containing about a $0.6 \mathrm{~mol}$ fraction of HEMA (see Figure 2). These results show that the property of the HEMA chain become the dominant factor in that of the block copolymer, suggesting that the contracted St chain is surrounded by the expanded HEMA chain in DMF-rich solvent.

The shape of the viscosity curve of the block

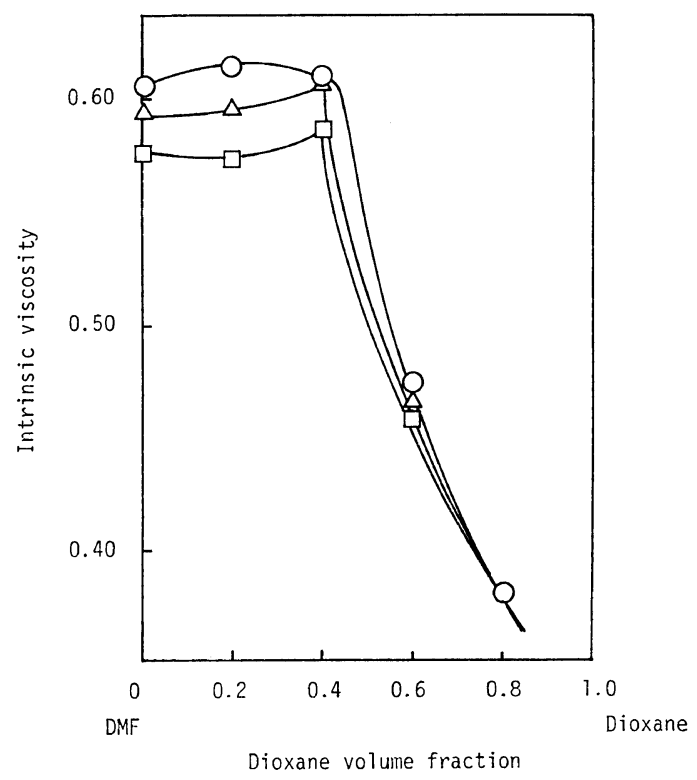

Figure 6. Relation between the intrinsic viscosity of the HEMA-St ABA-type block copolymer (HEMA mol fraction, 0.394) and the solvent composition in the $\mathrm{DMF}$-dioxane mixture: $\mathrm{O}$, at $15^{\circ} \mathrm{C}$; $\triangle$, at $30^{\circ} \mathrm{C}, \square$; at $50^{\circ} \mathrm{C}$. 
copolymer remarkably changed at temperatures between 15 and $30^{\circ} \mathrm{C}$. That is, with increasing temperature, the peak at about 0.2 disappeared, became a local minimum, and a cusp appeared at about 0.4. In consideration of the lesser compatibility between the HEMA chain and the St chain, the notable change in the shape of the viscosity curve with increasing temperature may suggest that the block copolymer adopts an intrachain phase-separated structure. However, as for the conformational change, i.e., the temperature dependence of the viscosity curves and the considerable decrease in the intrinsic viscosity beyond a 0.4 vol fraction, further detailed analyses must be carried out by other methods. Such studies are now in progress.

Figures 7 and 8 show the viscosity behavior of the random cooligomer and the block copolymer, respectively, in the ethanol-dioxane mixture. Ethanol is a good solvent for the HEMA chain, while a very poor one for the St chain. The random cooligomer with a HEMA mole fraction of 0.374 is insoluble in ethanol.

The viscosity curves for both the block copolymer and the random cooligomer possessed maxi-

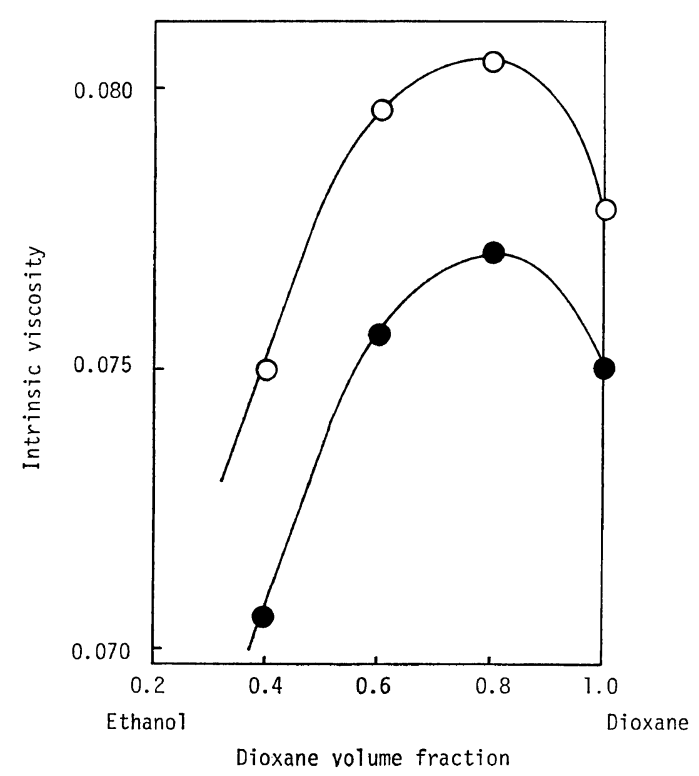

Figure 7. Relation between intrinsic viscosity of the HEMA - St random cooligomer (HEMA mol fraction, 0.374) and the solvent composition in the ethanoldioxane mixture: $\mathrm{O}$, at $30^{\circ} \mathrm{C} ; \bullet$, at $50^{\circ} \mathrm{C}$.

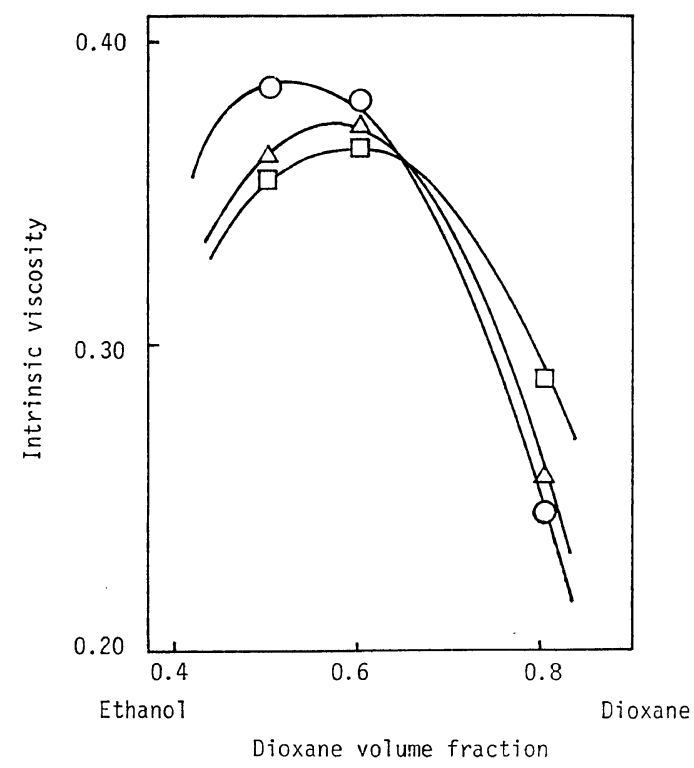

Figure 8. Relation between the intrinsic viscosity of the HEMA-St ABA-type block copolymer (HEMA mol fraction, 0.394) and the solvent composition in the ethanol-dioxane mixture: $O$, at $15^{\circ} \mathrm{C}$; $\triangle$, at $30^{\circ} \mathrm{C} ; \square$, at $50^{\circ} \mathrm{C}$.

mum peaks at optimum compositions of the solvent mixture. However, the temperature dependence of the peak position of the block copolymer was remarkably different from that of the random cooligomer. In the block copolymer system, the peak position shifted to the ethanol-rich composition in contrast to the random cooligomer which possessed a maximum at a constant composition, i.e., about a 0.8 vol fraction of dioxane. These peak shifts to the ethanolrich composition became large with decreasing temperature. It was found that the property of the HEMA chain became the dominant factor in that of the block copolymer; these contributions of the HEMA chain to the block copolymer became considerable with decreasing temperature in this solvent system as well as in the DMFdioxane system. However, the transition point of the intrinsic viscosity at relatively high temperature was not observed in the ethanol-dioxane system. It was considered that the viscosity behavior of the block copolymer was remarkably influenced by the selective solvent property. 


\section{T. OKano, M. IKemi, and I. Shinohara}

\section{REFERENCES}

1. See, for example, (a) M. Tollar, M. Stol, and K. Kliment, J. Biomed. Mater. Res., 3, 305 (1969); (b) F. J. Holly and M. F. Refojo, ibid., 9, 3061 (1975).

2. T. Okano, M. Katayama, and I. Shinohara, Nippon Kagaku Kaishi, 1908 (1976).

3. T. Okano, M. Katayama, S. Mogi, and I. Shinohara, Nippon Kagaku Kaishi, 88 (1977).

4. T. Okano, M. Katayama, and I. Shinohara, J. Appl. Polym. Sci., 22, 369 (1978).

5. T. Okano, M. Ikemi, and I. Shinohara, Nippon Kagakukaishi (J. Chem. Soc. Japan, Chem. Ind. Chem.), 93 (1977).

6. See, for example, (a) M. Girolamo and J. R. Urwin, Eur. Polym. J., 7, 693 (1971); (b) H.
Ohnuma, T. Kotaka, and H. Inagaki, Macromolecules, 7, 311 (1974); (c) A. Dondos, P. Rempp, and H. C. Benoit, Polymer, 13, 97 (1972); (d) idem., ibid., 16, 698 (1975).

7. H. Kawamura, K. Takemoto, and M. Inoue, Kobunshi Kagaku (Chem. High Polymers), 26, 148 (1969).

8. E. Tsuchida, I. Shinohara, and S. Kanbara, Kogyo Kagaku Zasshi (J. Chem. Soc. Japan, Ind. Chem. Sect.), 66, 329 (1963).

9. S. Siggia, J. G. Hanna, and I. R. Kervenski, Anal. Chem., 22, 1295 (1950).

10. G. Manecke and S. Singer, Makromol. Chem., 37, 119 (1966).

11) A. Dondos and D. Patterson, J. Polym. Sci., Part A-2, 7, 209 (1969). 